\title{
Instability dynamics of a horizontally shaken pendulum
}
Yan $\mathrm{Xu}^{1}$
Tristram J. Alexander ${ }^{2}$
Harvinder S. Sidhu ${ }^{3}$

(Received 18 January 2012; revised 2 July 2012)

\begin{abstract}
We consider the effect of horizontally shaking the pivot point of a damped pendulum. While similar systems have been considered in engineering in specific circumstances, for instance in the design of isolation systems in an earthquake zone, a careful analysis of the shaken damped pendulum behaviour has not been carried out. We identify the periodic solutions in the system, examine their bifurcation behaviour, and explore the development of chaos. We find that the horizontal shaking supports bistability in a critical frequency range and that the development of chaos depends sensitively on the driving frequency. Our work complements recent experiments on coupled pendulums with horizontal shaking, providing a foundation for further study of the pendulum dynamics.
\end{abstract}

http://journal.austms.org.au/ojs/index.php/ANZIAMJ/article/view/5153 gives this article, (C) Austral. Mathematical Soc. 2012. Published July 9, 2012. ISSN 1446-8735. (Print two pages per sheet of paper.) Copies of this article must not be made otherwise available on the internet; instead link directly to this URL for this article. 


\section{Contents}

1 Introduction

C326

2 Model

C327

3 System analysis

C328

4 Conclusion

C335

References

C338

\section{Introduction}

The damped-driven pendulum is a familiar object; the old-fashioned pendulum clock is a well-known example. However, it appears in a wide range of applications, from the operation of a crane [1] to an artificial heart [2]. Damped-driven pendulum models also form the basis of many theoretical investigations seeking to understand complex behaviour, from physical problems such as the nature of friction [3], to more general problems such as conditions for the emergence of chaos [4]. Pendulum-type models are widely used because they often capture the key dynamics of more complicated dynamical systems; however, often the details of the type of driving (and damping) vary between systems, and these differences have important effects.

The most well-known form of driving is that of an external oscillating force (for instance through a rotation of the pivot point of the pendulum), and chaotic motion is well known to appear in such a system [5, 6, e.g.]. Another commonly encountered driving force is that due to parametric driving, so named because a parameter of the system is varied with time (such as the length of the pendulum). Effective parametric driving through the vertical shaking of the pendulum pivot point has long been known [4, e.g.]. We instead consider the effect of horizontal shaking on the pendulum. Such 
driving is of particular interest from a theoretical perspective due to its possible decomposition in the limit of small pendulum oscillations into both a parametric component and an external driving force. Horizontal shaking has been recently examined in experiments on many pendulums [7], yet the underlying behaviour for a single pendulum is still largely unknown. However, related forms of such driving appear widely in engineering, for instance in pendulum isolation systems for response to seismic activity [8], but such driving has seen relatively little study from a nonlinear dynamics perspective. One notable exception is the finding that horizontal shaking may lead to the oscillation of the pendulum about a non-zero mean angle [9]. We examine in detail the bifurcation behaviour of the horizontally shaken pendulum and uncover the existence of bistability, intermittency and full chaotic dynamics.

\section{Model}

A schematic of the physical pendulum system we consider is shown in Figure 1 as adapted from the experimental setup considered by Thakur, English and Sievers [7] for a system of coupled pendulums. A rod of mass $m$ and length $L$ ending in a weight of mass $M$ is fixed on a torsional spring and suspended by a taut piano wire which is fastened on a frame. An electric motor is then used to drive the frame horizontally, so realizing the horizontal shaking of the pendulum. The equation of motion for the angle $\theta$ from vertical of the pendulum mass is, in dimensionless form,

$$
\ddot{\theta}+a \dot{\theta}+\sin (\theta)+f \omega^{2} \cos (\omega t) \cos (\theta)=0,
$$

where $a$ is the dimensionless damping coefficient, $f$ is the shaking amplitude $\omega=\omega_{d} / \omega_{0}$ is the ratio of the shaking frequency $\omega_{d}$ and the natural pendulum frequency $\omega_{0}$, and $t$ is in units of $\omega_{0}^{-1}$ (Thakur et al. [7] gave further details of the model). The effective torque on the pendulum mass depends on the angle $\theta$, leading to an effective parametric driving in the system. 


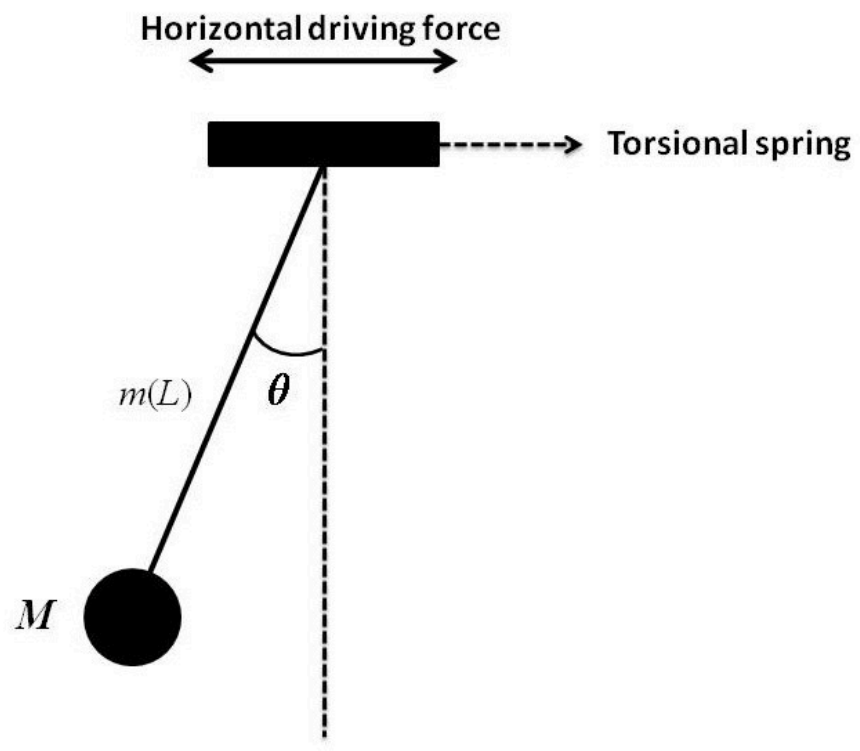

Figure 1: Sketch of model system. A mass $M$ at the end of a rod of length $L$ and mass $\mathrm{m}$, displaced an angle $\theta$ from the vertical and subjected to a horizontal driving force, exerted on the pendulum pivot.

\section{System analysis}

Our results are displayed using three main diagrammatic forms.

1. Trajectory plots: displaying the trajectory of a state variable such as the angle $\theta$ with time $t$.

2. Poincare sections: sampling the angle $\theta$ and angular velocity $\dot{\theta}$ at the driving frequency and plotting these values as points on a 2D graph.

3. Bifurcation diagrams: displaying the $\theta$ values only from the Poincare section, versus the changing control parameter $f$ (amplitude of driving). 
We begin our analysis by looking for harmonic solutions of the pendulum motion in the form $\theta=V_{c} \cos (\omega t+\phi)$, where $V_{c}$ is the amplitude of the pendulum oscillation and $\phi$ is the phase offset from the driving phase $\omega t$. We make two assumptions which simplify the problem. Firstly, we use Taylor series to replace the trigonometric nonlinearities with their lowest order algebraic nonlinear expansions; that is, $\sin \theta \approx \theta-\theta^{3} / 6$ and $\cos \theta \approx 1-\theta^{2} / 2$. Then we obtain the approximate model equation

$$
\ddot{\theta}+a \dot{\theta}+\left(\theta-\frac{1}{6} \theta^{3}\right)+f \omega^{2} \cos (\omega t)\left(1-\frac{1}{2} \theta^{2}\right)=0 .
$$

Secondly, we use the rotating wave approximation to neglect all third harmonic terms; that is, we assume $\cos ^{3}(\omega t+\phi) \mapsto 3 \cos (\omega t+\phi) / 4$. Making use of this second assumption we multiply Equation (2) by $\cos (\omega t)$ and integrate over one driving period $[0,2 \pi / \omega]$ to remove the dependence on $t$. This gives the following equation relating $V_{c}$ and $\phi$ :

$$
\begin{aligned}
& 8 V_{c} a \omega \sin \phi+2 \omega^{2} f V_{c}^{2} \cos ^{2} \phi+8 V_{c} \omega^{2} \cos \phi+V_{c}^{3} \cos \phi \\
& -8 V_{c} \cos \phi-8 f \omega^{2}+f \omega^{2} V_{c}^{2}=0 .
\end{aligned}
$$

Similarly multiplying Equation (2) by $\sin (\omega t)$ and performing the integration gives a second equation relating $V_{c}$ and $\phi$ :

$V_{c}\left(-8 \sin \phi+8 \omega^{2} \sin \phi+V_{c}^{2} \sin \phi+2 \omega^{2} f V_{c} \sin \phi \cos \phi-8 a \omega \cos \phi\right)=0$.

Setting $\boldsymbol{\omega}=0.8, \boldsymbol{a}=0.1$ and $\boldsymbol{f}=1.1$ we solve Equations (3) and (4) to obtain three real solutions for $\left(\mathrm{V}_{c}, \phi\right)$ : $(1.463,2.913),(1.662,0.293)$ and $(2.080,2.602)$. In the case of large $V_{c}$ the small $\theta$ approximation behind Equation (2) breaks down and we expect that our solutions will not be in good agreement with those of the original system (1). We compare the approximate solutions with those of the full model by numerically integrating the full system (1). With initial conditions $x(0)=0.5$ and $y(0)=0.1$ we obtain $V_{c}=0.8573$ and $\phi=5.0$ (see Figure 3), while for initial conditions $x(0)=1.0$ and $y(0)=0.5$ 
we converge to a solution with $V_{c}=2.07$ and $\phi=1.0$. We see that, as expected, there is only approximate agreement between the two sets of solutions. Significantly we find only two solutions through the numerical integration, and so we conclude that of the three solutions to (2) two are stable and one is unstable.

We examine the dependence of this bistability on $\omega$ in Figures $2-4$. We see that the bistability only exists for a range of $\omega$ (from approximately $\omega=0.6$ to $\omega=0.8$ ). Interestingly, we see that while the low amplitude solution is essentially out of phase with the shaking, the high amplitude solution is nearly in phase (see Figures 3 and 4 respectively: blue lines, $\theta(t)$; red lines, $\cos (\omega t)$ shaking). Comparing the analytical and numerical solutions over this range of $\omega$ we see reasonable agreement (see Figure 2), despite $V_{c}$ being large. The red stars are the solutions for the initial condition $x(0)=0.5$ and $y(0)=0.1$, and the green stars are the solutions for the initial condition $x(0)=-0.5$ and $y(0)=-2.0$.

We find that the system is sensitive to the initial conditions in the region of bistability. To expose this sensitivity we examine the basins of attraction for the two stable solutions in the range of initial conditions $\theta(0) \in[-\pi, \pi]$ and $\theta^{\prime}(0) \in[-2,2]$. The results are shown in Figure 5. The convergence to one or the other solution shows complex fine structure in the basins of attraction. But in general we see convergence predominantly to the low amplitude solution for low initial angles and predominantly to the high amplitude solution for large positive initial angles.

We now turn our attention to the behaviour of the pendulum as the forcing amplitude is varied. We examine the bifurcation behaviour of the pendulum dynamics in the range in which there are three solutions. Taking $\omega=0.7$, we find that for the given initial conditions $\left(\theta(0)=0.5\right.$ and $\left.\theta^{\prime}(0)=0.1\right)$ the pendulum converges to one periodic solution as the forcing amplitude $f$ is varied up to $f \approx 5$, as shown in Figure 6(a). For $f>5$ the pendulum exhibits complex windows of chaotic motion and periodicity. Increasing the frequency to $\omega=0.8$, we obtain the more complex bifurcation behaviour 


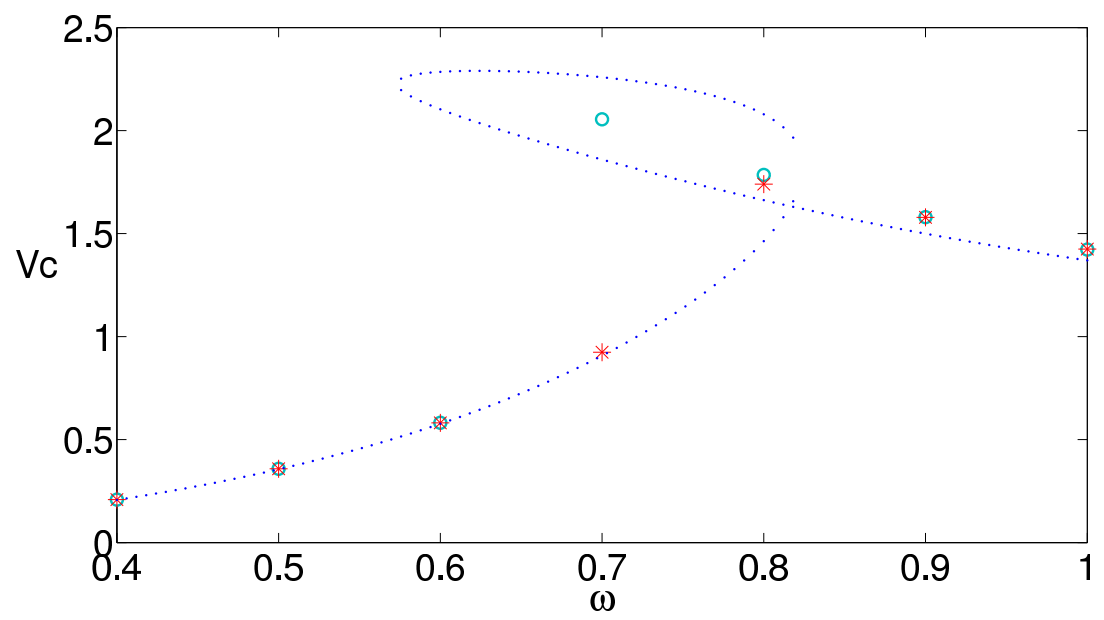

Figure 2: Comparison between solutions of the approximate model (2) and numerical solutions of the full model (1): the small blue dots are the solutions to (2); the blue circles and red stars are two sets of numerical solutions to system (1) (found under different initial conditions: blue, initial conditions $x(0)=0.5$ and $y(0)=0.1$; red, initial conditions $x(0)=-1.5$ and $y(0)=$ $-2.0)$.

at small values of $f$, as shown in Figure $6(\mathrm{~b})$. For $f<1.2$ two periodic solutions are possible, and the bifurcation plot of Figure 6(b) shows jumps between two values as a consequence of this. At $f=1.2$ chaotic behaviour begins to appear (noticeable by the stream of vertical points appearing in the bifurcation diagram) before the dynamics settle down to periodic behaviour (Figure 7). The intervals of chaos become steadily longer until at $f=1.45$ no periodic behaviour is seen (Figure 8(a)). The solution becomes regular again for $f>1.6$ over a large parameter window (Figure $6(b)$ ). In this region the dynamics correspond to the periodic oscillation of the pendulum.

The chaotic motion is visualised using the Poincare map. We plot the values of $\theta$ and $\theta^{\prime}$ every $2 \pi / \omega$ timestep as points on the graph. When the dynamics are chaotic the points trace out the chaotic attractor shown in Figure 8(b). 


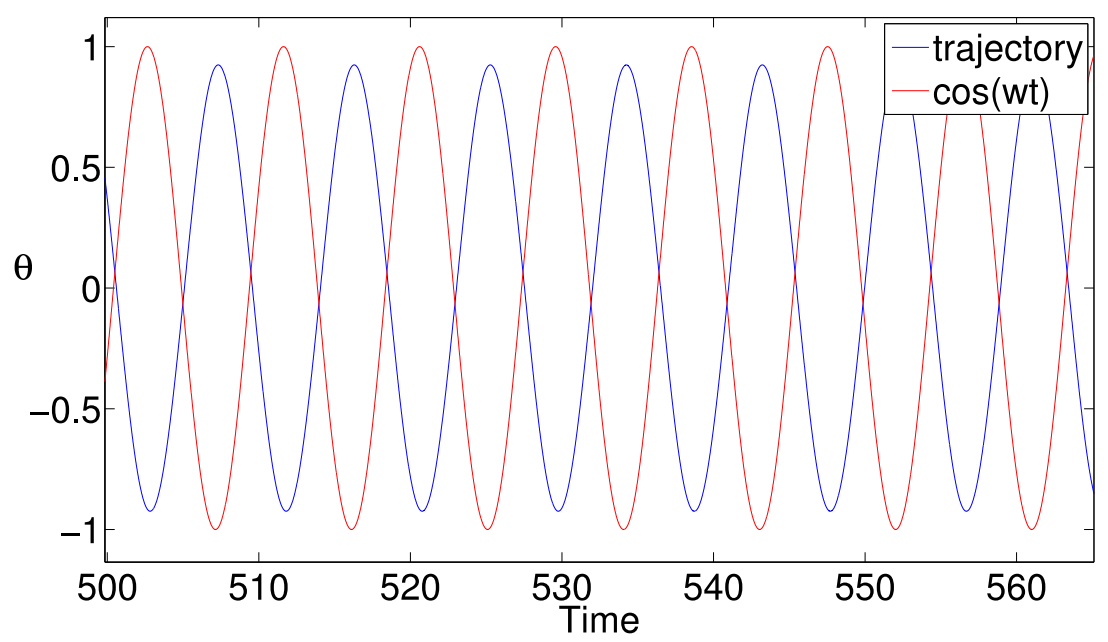

Figure 3: Trajectory for initial condition $x(0)=0.5$ and $y(0)=0.1$ when $\omega=0.7, a=0.1$ and $f=1.1$.

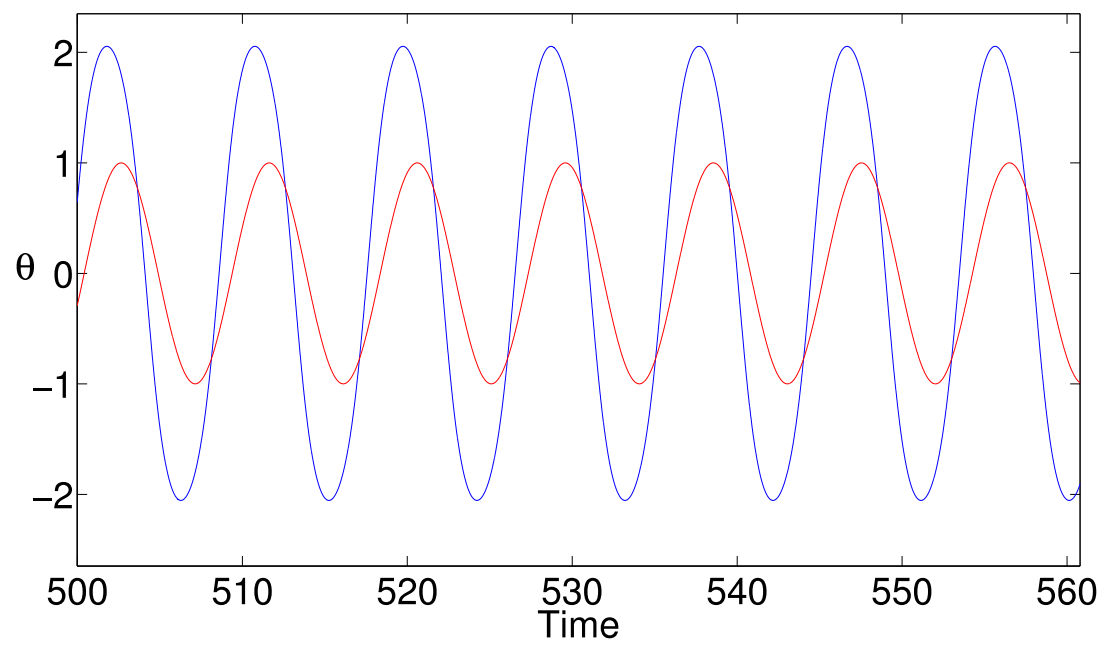

Figure 4: Trajectory for the initial condition $x(0)=-0.5$ and $y(0)=-2.0$ when $\omega=0.7, a=0.1$ and $f=1.1$. 


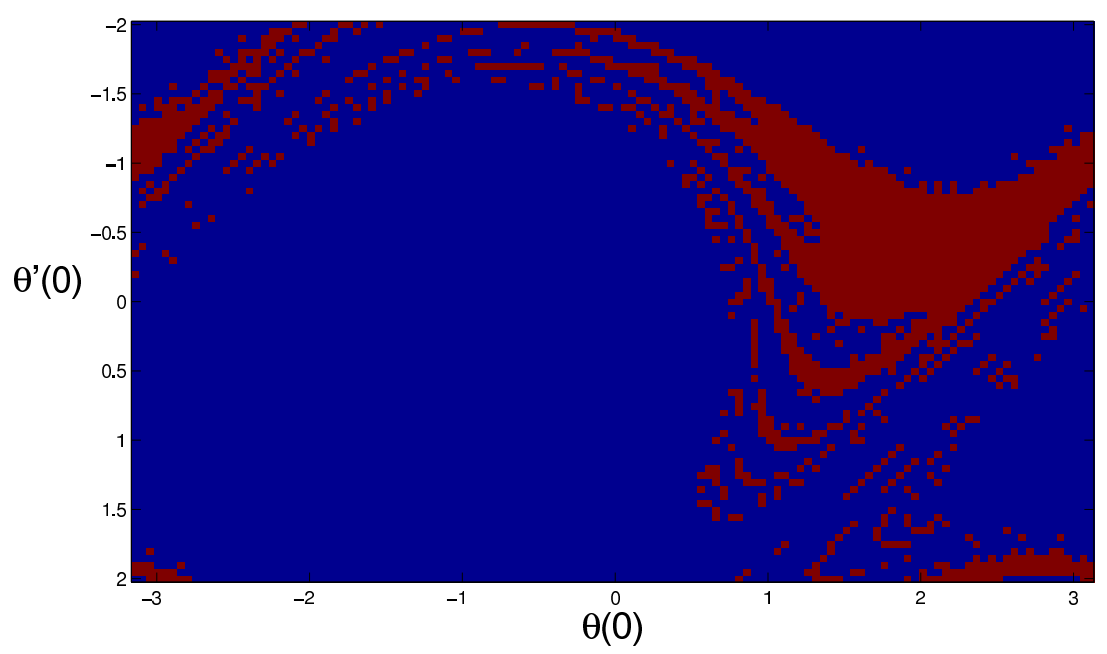

Figure 5: The basins of attraction for the two different periodic solutions at $a=0.1, f=1.1$ and $\omega=0.7$. Blue and red correspond to small and large amplitude solutions respectively.

This attractor appears even when the chaotic motion eventually settles to a periodic state, due to the initial chaotic transient. We made use of the periodicity of $\theta$ and mapped all values of $\theta$ into the domain $\theta \in[0,2 \pi]$.

Lastly, we examine the dynamics after the first chaotic interval in the bifurcation diagram. Figure 9 shows an example periodic trajectory (when $a=0.1$, $\omega=0.8$ and $f=1.8$ ), suggesting that the dynamics become regular for larger driving amplitude. However, we find that for some initial conditions and parameter values the pendulum may instead converge to a steadily rotating state. These results indicate that the full picture of the dynamics of the damped-driven pendulum is highly complex, hinting at further bifurcations which are beyond the scope of this present work. Such complexity is expected given that the more well studied torque-driven pendulum exhibits similarly complex bifurcation behaviour. 


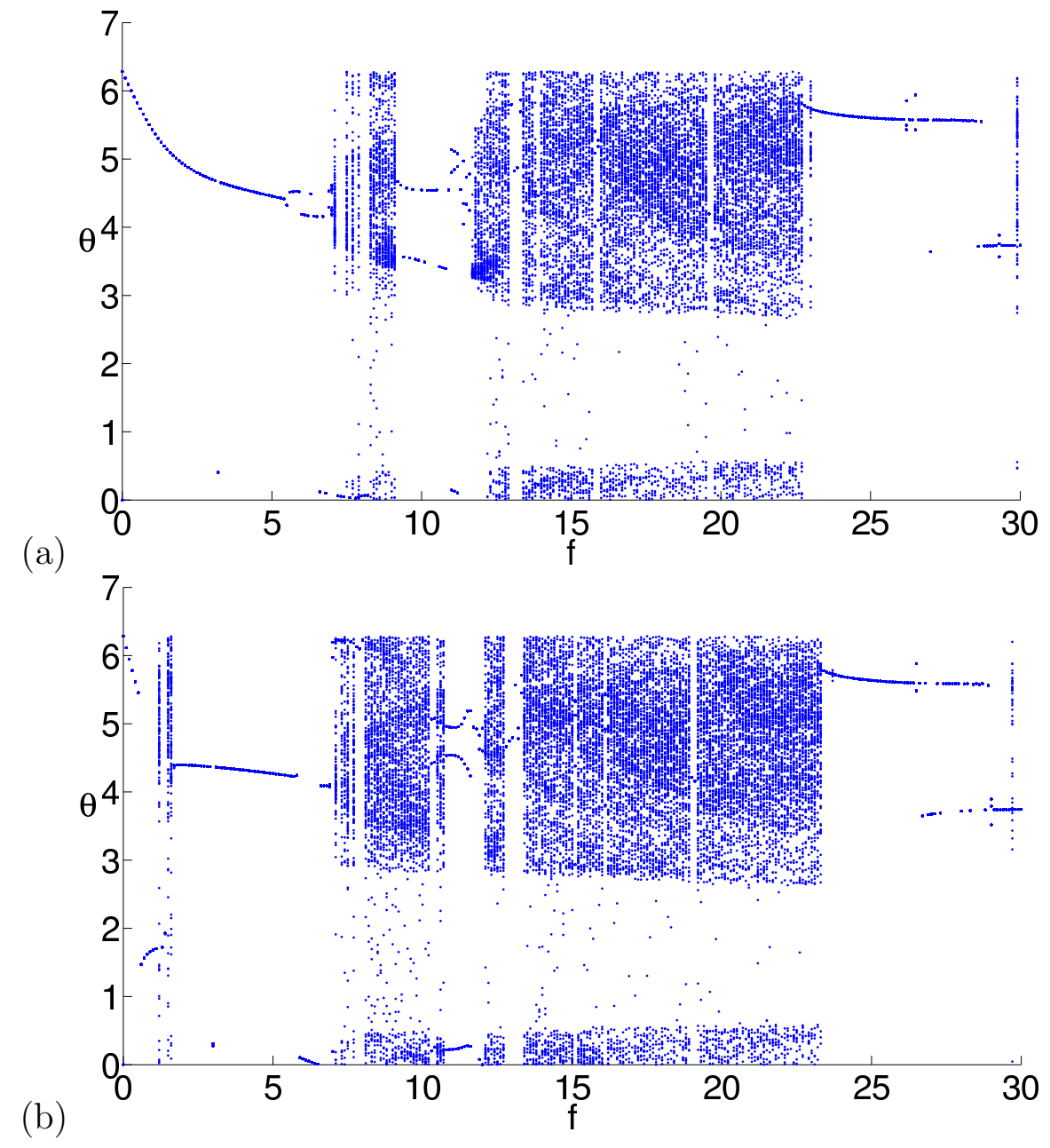

Figure 6: (a) The bifurcation diagram for $a=0.1, f=1.1$ and $\omega=0.7$. (b) The bifurcation diagram for $a=0.1, f=1.1$ and $\omega=0.8$. In both cases the initial conditions used are $\theta(0)=0.5$ and $\theta^{\prime}(0)=0.1$. The diagram is constructed by plotting the $\theta$ values every $t=2 \pi / \omega$ timestep after an initial period of $t=1000$ to remove initial transient behaviour. 


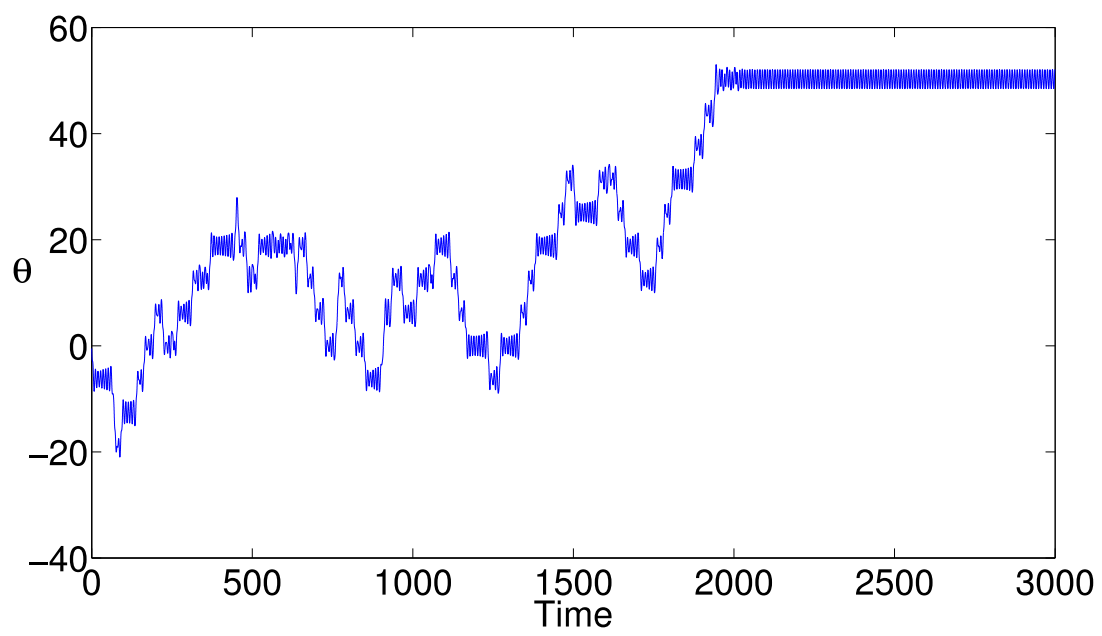

Figure 7: A trajectory demonstrating the intermittent route to chaos $(a=0.1$, $\omega=0.8$ and $f=1.2$ ).

\section{Conclusion}

Our work uncovers some of the complex behaviour found in a horizontally shaken pendulum. We find that the horizontal shaking supports bistability in a critical frequency range, with the two possible periodic solutions corresponding to low and high amplitude oscillations which are out-of-phase and approximately in-phase with the shaking, respectively. We find that the system exhibits an interesting form of intermittency on the route to chaotic behaviour, displaying increasingly longer periods of chaotic behaviour before settling into periodic motion. The development of this chaotic behaviour depends critically on the ratio of the driving and natural frequencies of the pendulum, where we have examined the case in which the driving frequency is lower than the natural frequency. For larger shaking amplitudes the pendulum returns to periodic behaviour; however, it may also exhibit rotations for certain initial conditions and parameter values, hinting at the further complexity of this driven system. Our work opens up the possibility of further study on 


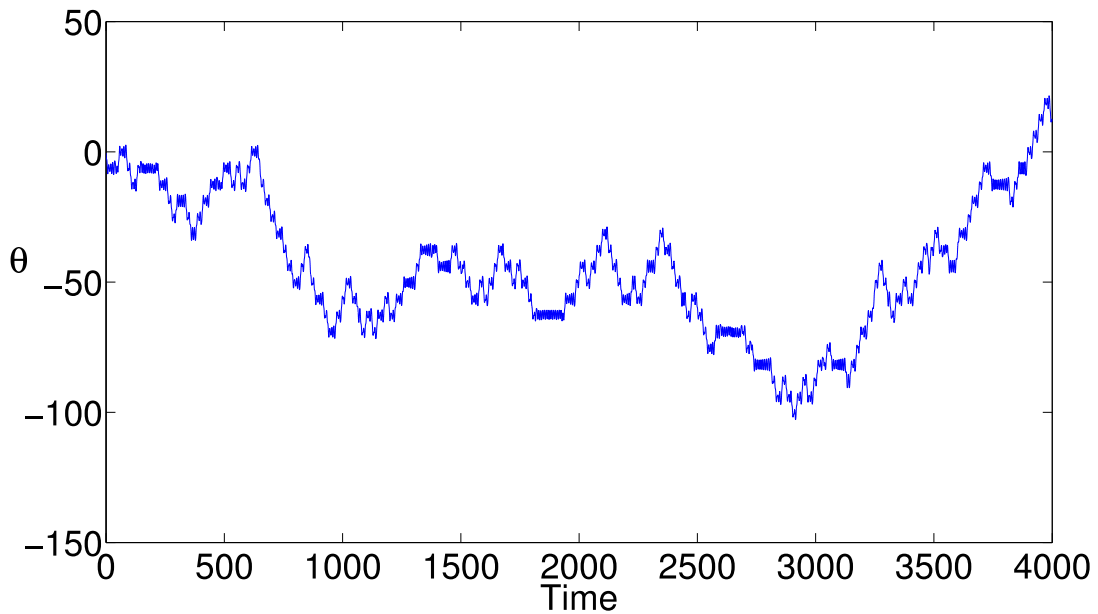

(a)

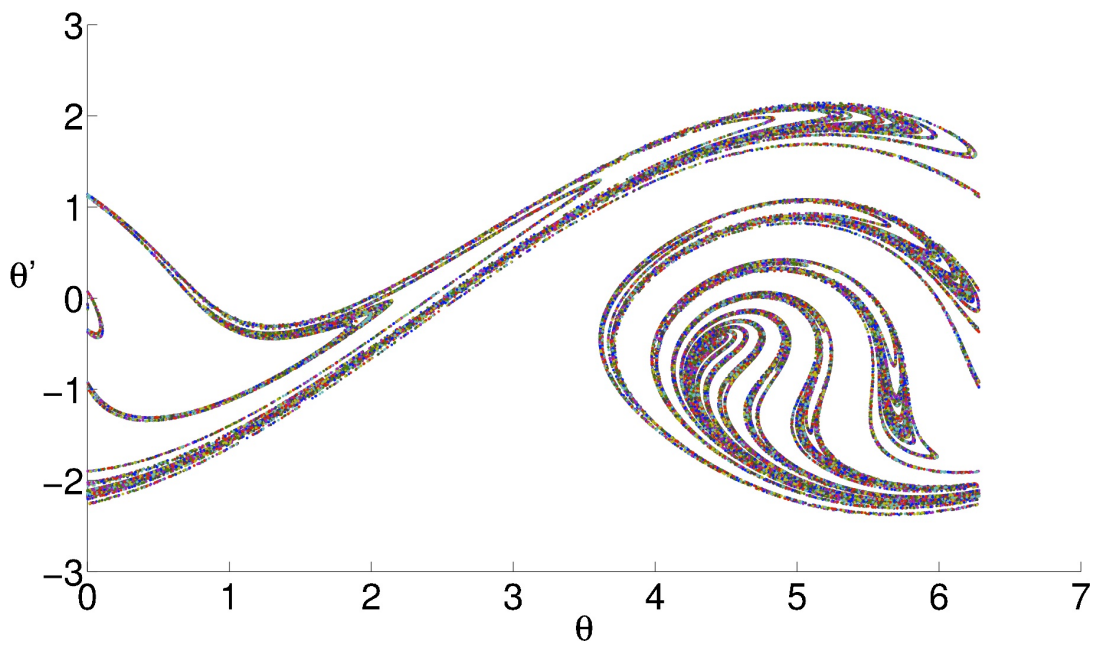

Figure 8: (a) A trajectory showing fully developed chaos $(a=0.1, \omega=0.8$ and $f=1.45$ ). (b) The Poincare section of developed chaos when $a=0.1$, $\omega=0.8$ and $f=1.45$. 


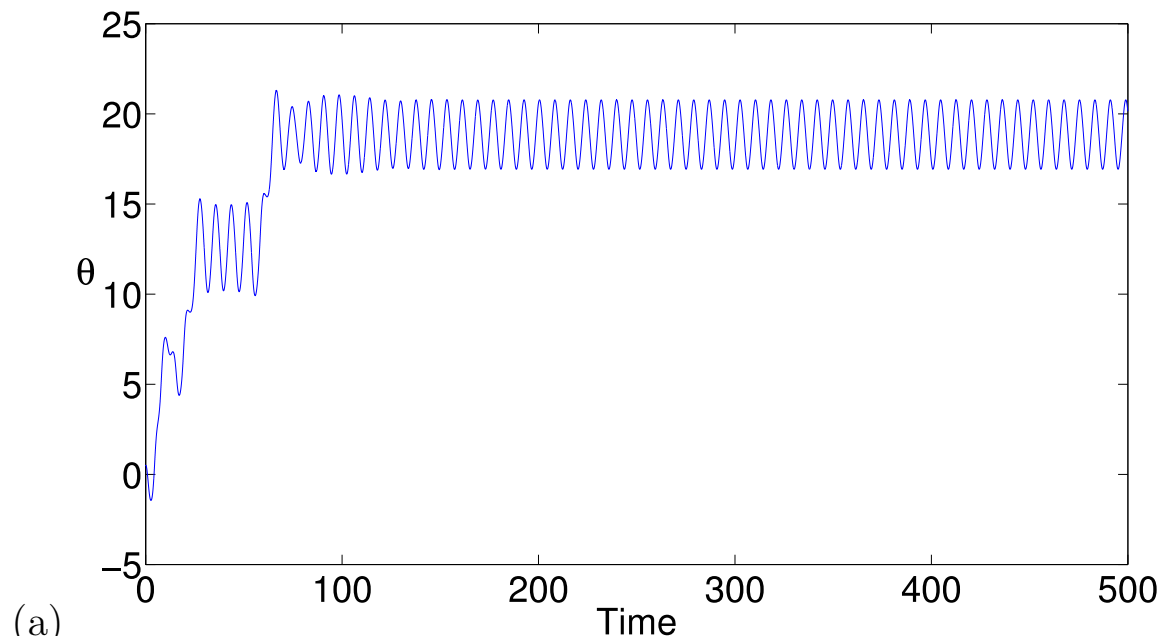

(a)

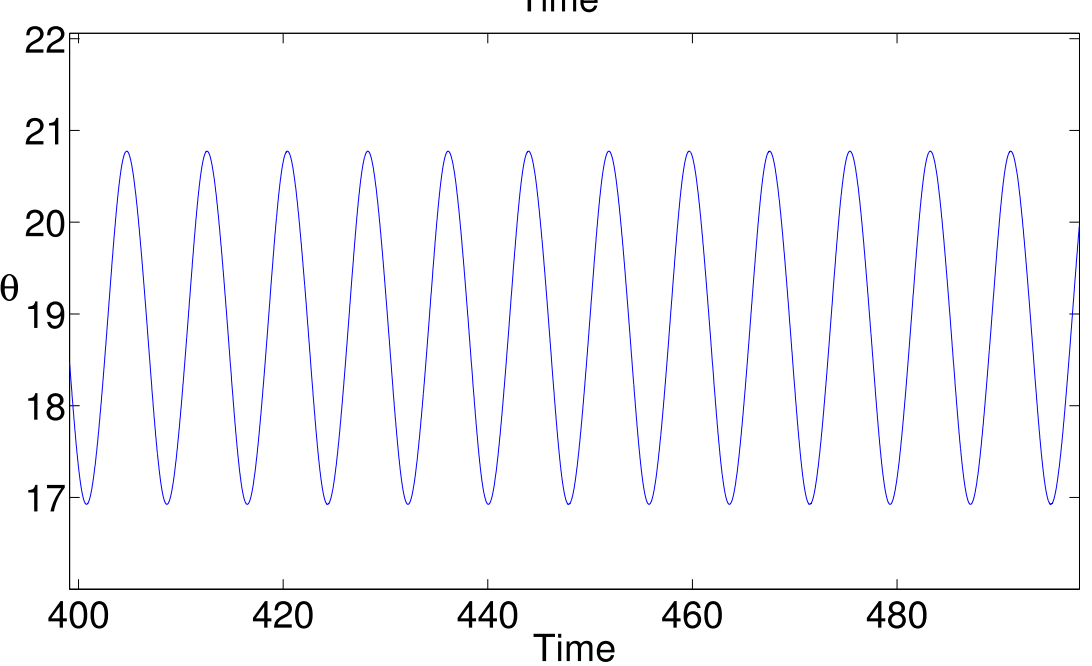

(b)

Figure 9: (a) An example trajectory from beyond the chaotic region in parameter space, at $a=0.1, w=0.8$ and $f=1.8$. (b) A magnification of the stable evolution. 
this system and our future work will be extended to the theoretical analysis of the more complex instabilities which have been observed in experiments on shaken pendulum chains [7].

Acknowledgements We express appreciation to the people who helped us in the research. We thank UNSW@ADFA for providing an excellent working environment. Especially we thank the finance support from the China Scholarship Council and UNSW@ADFA.

\section{References}

[1] Z. Masoud, A. Nayfeh and D. Mook, Cargo pendulation reduction of ship-mounted cranes. Nonlinear Dynamics, 35, 2004, 299-311. doi:10.1023/B:NODY.0000027917.37103.bc C326

[2] I. M. Sauer, J. Frank and E. S. Bücherl, Proposal of a new electromechanical total artificial heart: the TAH Serpentina. Artifical Organs, 23, 1999, 290-291. doi:10.1046/j.1525-1594.1999.06226.x C326

[3] A. Mokha, M. C. Constantinon, A. M. Reinhorn, et al. Experimental study of friction-pendulum isolation system. Journal of Structural Engineering, 117(4), 1991, 1201-1217. doi:10.1061/(ASCE)0733-9445(1991)117:4(1201) C326

[4] R. W. Leven, B. P. Koch, Chaotic behaviour of paramentrically excited damped pendulum. Physics Letters A, 86(2), 1981, 71-74. doi:10.1016/0375-9601(81)90167-5 C326

[5] H. Heng, W. Martienssen, Analysing the chaotic motion of a driven pendulum. Chaos, Solitons and Fractals, 2(3), 1992, 323-334. doi:10.1016/0960-0779(92)90039-P C326 
[6] G. L. Baker, Control of the chaotic driven pendulum. American Journal of Physics, 63(9), 1995, 832-838. doi:10.1103/PhysRevLett.74.1974 C326

[7] R. B. Thakur, L. Q. English and A. J. Sievers, Driven intrinsic localized modes in a coupled pendulum array. Journal of Physics: Applied Physics, 41, 2008, 015503. doi:10.1088/0022-3727/41/1/015503 C327, C338

[8] Y. Nakamura, M. Saruta, A. Wada, T. Takeuchi, S. Hikone and T. Takahashi, Development of the core-suspended isolation system, Earthquake Engineering \& Structural Dynamics 40, 2011, 429-447. doi:10.1002/eqe.1036 C327

[9] J. M. Schmitt, P. V. Bayly, Bifurcations in the mean angle of a horizontally shaken pendulum: analysis and experiment. Nonlinear Dynamics, 15, 1998, 1-14. doi:10.1023/A:1008279910762 C327

\section{Author addresses}

1. Yan Xu, Applied and Industrial Mathematics Research Group, School of Physical, Environmental and Mathematical Sciences, UNSW@ADFA, Canberra, ACT 2610, Australia. mailto:yan. xu@student.adfa.edu. au

2. Tristram J. Alexander, Applied and Industrial Mathematics Research Group, School of Physical, Environmental and Mathematical Sciences, UNSW@ADFA, Canberra, ACT 2610, Australia. mailto:t.alexander@adfa.edu.au

3. Harvinder S. Sidhu, Applied and Industrial Mathematics Research Group, School of Physical, Environmental and Mathematical Sciences, UNSW@ADFA, Canberra, ACT 2610, Australia.

mailto:h.sidhu@adfa.edu.au 